\title{
Letter \\ $10 \%$ Hydroxyethylstarch impairs renal function and induces interstitial proliferation, macrophage infiltration and tubular damage
}

\author{
Martin Siegemund
}

Department of Anaesthesia and Intensive Care Medicine, Surgical ICU, University Hospital Basel, Surgical ICU, Spitalstrasse 21, CH-4031 Basel, Switzerland

Corresponding author: Martin Siegemund, siegemundm@uhbs.ch

Published: 8 July 2009

Critical Care 2009, 13:413 (doi:10.1186/cc7928)

This article is online at http://ccforum.com/content/13/4/413

(c) 2009 BioMed Central Ltd

See related research by Hüter et al., http://ccforum.com/content/13/1/R23

Hüter and colleagues recently published an experimental paper about possible pathomechanisms of hydroxyethylstarch (HES)-induced adverse effects on renal function in an isolated perfusion model of 6 hours [1]. The authors should be congratulated for their attempt to shed light on the influence of different HES preparations on renal function, combining functional results and histological data.

In the recently published prospective, randomized, controlled Efficacy of Volume Substitution and Insulin Therapy in Severe Sepsis (VISEP) trial, 10\% HES 200/05 caused a close to significant increase in 90-day mortality in septic patients. Renal failure and renal replacement therapy significantly increased dose dependently compared with Ringer's lactate treatment. Unfortunately, 100 out of 262 patients in the HES group received more than the maximum allowed daily dose on at least 1 day, the majority occurring on the first day after study inclusion. The patients without a violation of the maximum daily dose administration had a mortality rate even lower than that in the Ringer's lactate group [2].

In their isolated renal perfusion model, Hüter and colleagues tried to answer some of the questions originating from the VISEP trial, comparing 10\% HES 200/0.5, 6\% HES 130/0.42 and Ringer's lactate [1]. The hyperoncotic 10\% HES, used in the VISEP study, showed severe oliguria, impaired potassium excretion and signs of lysosomal tubular damage. In contrast, isovolemic 6\% HES showed no difference compared with Ringer's lactate in creatinine clearance, sodium excretion and $N$-acetyl- $\beta$-D-glucosamidase in urine. The $6 \%$ HES even showed a decreased inflammatory reaction compared with $10 \%$ HES and Ringer's lactate.
Interestingly, osmotic-nephrosis-like lesions were found in all three groups, also to a lesser extent in the Ringer's lactate group. These lesions represent a quantity-dependent accumulation of proximal tubular lysosomes due to administration of exogenous solutes [3]. Principally, the lysosomal swelling is reversible, but any process such as ischaemia or pre-existing kidney damage that impairs lysosomal digestion further delays degradation. The presence of osmotic nephrosis does not necessarily have an impact on proximal tubular function [3].

The second significant difference between 6\% HES and Ringer's lactate was the amount of urine produced [1]. The authors take this together with the increased amount of osmotic nephrosis as a sign of impaired renal function due to $6 \%$ HES. An alternative explanation for the difference in urinary output would be $0.9 \% \mathrm{NaCl}$, the carrier fluid of the HES solution. In an elegant animal model, Wilcox showed that hyperosmolar chloride-containing solutes reduce renal blood flow, the glomerular filtration rate, urinary output and sodium reabsorption [4]. The depression of renal function was even increased in hypovolemic, potassium-depleted animals. Healthy young human volunteers showed a lower urinary output, a longer time to first micturition and decreased urinary sodium after infusion of 2 I of $0.9 \% \mathrm{NaCl}$ compared with Hartman's solution [5].

In my opinion, the study by Hüter and colleagues experimentally confirms the data from the VISEP study showing that hyperoncotic $10 \%$ HES is nephrotoxic. They should not extend their statement to isooncotic 6\% HES because in their study they only found small differences from Ringer's lactate - differences with debatable clinical relevance or differences ascribable to saline. 


\section{Author's response \\ Lars Hüter, Tim-Philipp Simon, Lenard Weinmann, Tobias Schürholz, Konrad Reinhart, Gunter Wolf, Kerstin Ute Amann and Gernot Marx}

Siegemund pointed out in his letter that according to our study results 10\% HES is nephrotoxic while 6\% HES 130/0.42 is comparable with Ringer's lactate in its effects. Siegemund also suggested in his letter that this can be explained by the hyperviscosity of $10 \%$ HES $200 / 0.5$ and the solvent natural saline [1].

We agree that a hyperviscous solution may by itself aggravate renal impairment. This adds further arguments against the use of $10 \%$ HES solutions. We used $10 \%$ HES $200 / 0.5$ solution in a dosage that corresponds to $100 \%$ of the maximal daily dosage and used 6\% HES 130/0.42 solution in a dosage corresponding to $66 \%$ of the maximal daily dosage in humans. These dosages resulted in a comparable amount of $2 \mathrm{~g} / \mathrm{kg}$ of both HES solutions. We therefore agree with Siegemund's comments that in our study we found differences between 10\% HES 200/0.5 and 6\% HES 130/0.42.

The concept of balanced solutions is currently widely discussed. In experimental septic shock a balanced HES preparation resulted in significant improved short-term survival as compared with a saline-based resuscitation [6]. In our study, however, both starches were solved in saline. The suggested nephrotoxicity of saline in our model therefore needs to be investigated in another study.

In our view, Siegemund drew a false conclusion from the VISEP study results - claiming that 'The patients without a violation of the maximum daily dose administration had a mortality rate even lower than that in the Ringer's lactate group'. This is an incorrect comparison, because it is not possible to compare the mortality rate of one post-hoc subgroup with the mortality rate of the overall crystalloid group. The VISEP study showed that patients receiving $10 \%$ HES 200/0.5 had no improved outcome over patients receiving Ringer's lactate at 28 days. Moreover, subgroup analysis found that patients who had received a lower dose of $10 \%$ HES 200/0.5 also were more likely to have renal failure than those who had received Ringer's lactate $(30.9 \%$ vs. $21.7 \%, P=0.04)$ and were more likely to need renal replacement therapy (25.9\% vs. $17.3 \%, P=0.03)$ [2].

\section{Competing interests}

The author declares that they have no competing interests.

\section{References}

1. Hüter L, Simon TP, Weinmann L, Schürholz T, Reinhart K, Wolf G, Amann KU, Marx G: Hydroxyethylstarch impairs renal function and induces interstitial proliferation, macrophage infiltration and tubular damage in an isolated renal perfusion model. Crit Care 2009, 13:R23.

2. Brunkhorst FM, Engel C, Bloos F, Meier-Hellmann A, Ragaller M, Weiler N, Moerer O, Gruendling M, Oppert M, Grond S, Olthoff D, Jaschinski U, John S, Rossaint R, Welte T, Schaefer M, Kern P, Kuhnt E, Kientopf M, Hartog C, Natanson C, Loeffler M, Reinhart K, for the German Competence Network Sepsis (SepNet): Intensive insulin therapy and pentastarch resuscitation in severe sepsis. N Engl J Med 2008, 358:125-139.

3. Dickenmann M, Oettl T, Mihatsch MJ: Osmotic nephrosis: acute kidney injury with accumulation of proximal tubular lysosomes due to administration of exogenous solutes. Am J Kidney Dis 2008, 51:491-503.

4. Wilcox CS: Regulation of renal blood flow by plasma chloride. J Clin Invest 1983, 71:726-735.

5. Reid F, Lobo DN, Williams RN, Rowlands BJ, Allison SP: (Ab)Normal saline and physiological Hartmann's solution: a randomized double-blind crossover study. Clin Sci (Lond) 2003, 104:17-24.

6. Kellum JA: Fluid resuscitation and hyperchloremic acidosis in experimental sepsis: improved short-term survival and acidbase balance with Hextend compared with saline. Crit Care Med 2002, 30:300-305. 